\title{
Mechanically functional amyloid fibrils in the adhesive of a marine invertebrate as revealed by Raman spectroscopy and atomic force microscopy
}

\author{
Anika S. Mostaert ${ }^{1,2}$, Rowena Crockett ${ }^{3}$, Graham Kearn ${ }^{4}$, Izhack Cherny ${ }^{5 *}$, Ehud Gazit ${ }^{5}$, \\ Louise C. Serpell ${ }^{6}$, and Suzanne P. Jarvis ${ }^{2}$ \\ ${ }^{1}$ School of Biology and Environmental Science, University College Dublin, Dublin, Ireland; ${ }^{2}$ Conway Institute of \\ Biomolecular and Biomedical Research, University College Dublin, Dublin, Ireland; ${ }^{3}$ Swiss Federal Laboratories for \\ Material Testing and Research, Duebendorf, Switzerland; ${ }^{4}$ School of Biological Sciences, University of East Anglia, \\ Norwich, Norfolk, UK; ${ }^{5}$ Department of Molecular Microbiology and Biotechnology, Tel Aviv University, Tel Aviv, \\ Israel; and ${ }^{6}$ Department of Biochemistry, University of Sussex, Falmer, UK
}

\begin{abstract}
Summary. Amyloid fibrils are primarily known in a pathogenic context for their association with a wide range of debilitating human diseases. Here we show a marine invertebrate (Entobdella soleae) utilizes functional amyloid fibrils comparable to those of a unicellular prokaryote (Escherichia coli). Thioflavin-T binding and Raman spectroscopy provided evidence for the presence of amyloid in the adhesive of Entobdella soleae. We elucidated that for these two very different organisms, amyloid fibrils provide adhesive and cohesive strength to their natural adhesives. Comparing the nanoscale mechanical responses of these fibrils with those of pathogenic amyloid by atomic force microscopy revealed that the molecular level origin of the cohesive strength was associated with the generic intermolecular $\beta$-sheet structure of amyloid fibrils.
\end{abstract}

Received December 10, 2008

* Present address of I.C.: Department of Chemistry, Princeton University, NJ 08540, USA

Address for correspondence: Dr. Anika Mostaert, Conway Institute, University College Dublin, Dublin 4, Ireland.

Tel: +353-1-7166766, Fax: +353-1-7166701

E-mail: anika.mostaert@ucd.ie

or Prof. Suzi Jarvis, Conway Institute, University College

Dublin, Dublin 4, Ireland

Tel: +353-1-7166780, Fax: +353-1-7166777

E-mail: suzi.jarvis@ucd.ie
Functional adhesive residues were found only in the case of the functional amyloid. Atomic force microscopy provided a useful means to characterize the internal structural forces within individual amyloid fibrils and how these relate to the mechanical performance of both functional and pathogenic amyloid. The mechanistic link of amyloidbased cohesive and adhesive strength could be widespread amongst natural adhesives, irrespective of environment, providing a new strategy for biomimicry and a new source of materials for understanding the formation and stability of amyloid fibrils more generally.

\section{Introduction}

Nature has evolved many extraordinary adhesive strategies that are essential for the survival of a wide diversity of micro- and macro-organisms in marine, freshwater and terrestrial habitats. A large amount of work on natural adhesives has focused on marine organisms in order to prevent biofouling, or to develop aqueous-based biomimetic adhesives (Kamino, 2008). These adhesives vary in that a sessile marine organism depends upon permanent adhesion, but motile organisms rely on transitory adhesion during locomotion, or temporary adhesion to different surfaces (Walker, 1987).

While research in this area progresses, no common underlying strategy has emerged in natural adhesives. For example, attachment of the blue mussel is reliant upon an adhesive with a high content of 3,4-dihydroxy- 
L-phenylalanine (DOPA) (Waite and Tanzer, 1981; Waite, 1983), whereas the dry, temporary adhesion of the Tokay gecko is attributed to weak van der Waals forces between a surface and the fine setae on gecko toes (Autumn et al., 2000., 2002). The merging of these two adhesion strategies has recently produced a superior biomimetic hybrid adhesive (Lee et al., 2007). Here we study the temporary, marine-based adhesive of a fish skin parasite (Entobdella soleae) and a bacterial (Escherichia coli) biofilm adhesive, and find a surprising structural commonality between these two otherwise unconnected natural adhesives.

Entobdella soleae (Monogenea: Capsalidae) is a parasitic marine flatworm that firmly attaches to the skin of the common sole (Solea solea) (Kearn and EvansGowing, 1998). The parasite moves like a leech, using anterior pads on the head for temporary attachment, while the posterior attachment organ (the haptor) is detached and relocated (Kearn and Evans-Gowing, 1998). These pads attach not by suction, but by means of an adhesive secretion. This tissue adhesive is of intrinsic interest, not only because of its critical role in the parasite's establishment and subsequent survival, but also as it works rapidly to provide strong adhesion to wet surfaces (Kearn and Evans-Gowing, 1998). Previous chemical characterisation of this adhesive has shown that it does not contain lipid, polysaccharide or DOPA, but instead consists of a network of highly insoluble proteinaceous fibres (Hamwood et al., 2002) .

The fibres bare a similar morphology to curli, the proteinacious fibrils in the natural adhesive expressed by Escherichia coli. These extracellular adhesive fibres have been identified as amyloid fibrils using congo red and thioflavin T binding (Chapman et al., 2002), and their primary function is to promote colonization of a surface (Olsen et al., 1989). Surface-associated amyloid fibrils have since been described in the biofilms of bacteria within several phyla (Larsen et al., 2007; Otzen and Nielsen, 2007). Amyloid fibrils are primarily known for their association with a wide range of degenerative human diseases, such as Alzheimer's and Parkinson's diseases (Lansbury, 1999). However, in recent years the identification of amyloid in a range of functional roles has emerged (Chiti and Dobson, 2006; Mostaert et al., 2006; Fowler et al., 2007; Cherny and Gazit, 2008).

The aim of this study was to investigate if the adhesive fibres of Entobdella soleae also have the generic amyloid core structure of an intermolecular $\beta$-sheet. Here we characterize the mechanical properties of the adhesive fibrils extruded from the parasite with atomic force microscopy (AFM), and compare these data to those obtained for the amyloid-based adhesive from E. coli.
Employing Raman spectroscopy and histochemical staining with AFM allowed both physical and chemical properties of the adhesive protein structures of Entobdella soleae to be made in situ. If the adhesive of this marine parasite is indeed amyloid-based, this would provide the first mechanistic link between two otherwise unrelated natural adhesives.

\section{Materials and Methods}

\section{Pathogenic fibril preparation}

Pre-formed fibrils of recombinant human $\alpha$-synuclein were provided by Dr Shahin Zibaee (Cambridge University, UK), and were prepared according to Zibaee et al. (2007). For the Raman spectroscopy, A $\beta_{25-35}$ fibrils were grown from a synthesized amyloid $\beta$-Protein $25-35$ (H-1192, Bachem, UK). This peptide was selected due to its ability to form fibrils readily in $\mathrm{H}_{2} \mathrm{O}$ (Yanker $e t$ al., 1990), providing a means for deuterium exchange during the formation process. The peptide was dissolved in milliQ ultrapure water (Millipore, MA, USA) or $100 \% \mathrm{D}_{2} \mathrm{O}$ (Sigma, Switzerland) at a concentration of approximately $1 \mathrm{mM}$. Fibrils formed within one week at room temperature (verified by Congo red birefringence, thioflavin-T binding and AFM imaging).

\section{Secreted functional fibrils}

Curli fibrils were obtained from E. coli MC4100, the wild-type curli producing strain. The curli attachment method is based on that described previously (Pawar et al., 2005). Diluted MC4100 culture (1:100) was placed into a polypropylene falcon together with a glass coverslip. The cells were allowed to attach to the glass surface at $28^{\circ} \mathrm{C}$ for seven days with slow orbital shaking $(\sim 50 \mathrm{rpm})$. The coverslip was then removed, and stored in a sealed Petri dish to keep fibrils hydrated until analysis. Specimens of Entobdella soleae were maintained in aquaria as parasites on the skin of Solea solea. The secreted adhesive was collected by manually manipulating live parasites to extrude adhesive 'prints' from their anterior end onto a clean glass slide. This procedure is described in full elsewhere (Hamwood et al., 2002).

\section{Thioflavin T binding}

Binding of the benzothiazole dye thioflavin-T (SigmaAldrich, Ireland) to amyloid structures was detected by directly staining samples on glass slides with $10 \mu \mathrm{m}$ 
aqueous thioflavin-T for five minutes, washing in milliQ water, and viewing immediately at $63 \mathrm{X}$ magnification with a Zeiss Confocal META system microscope (LSM 510, Carl Zeiss Ltd, UK). Emission of thioflavin-T dye binding to amyloid was previously determined (Mostaert et al., 2006) as a peak around $550 \mathrm{~nm}$ after excitation at $488 \mathrm{~nm}$ and emission collected at 505 to $620 \mathrm{~nm}$ using a control sample of amyloid fibrils grown from $\mathrm{A} \beta_{25-35}(\mathrm{H}-$ 1192, Bachem, UK). An unstained A $\beta_{25-35}$ amyloid fibril control emitted no fluorescence signal when excited the same way as the thioflavin-T stained control.

\section{Raman spectroscopy}

Raman spectra were collected with a Renishaw MicroRaman (Renishaw, UK) with a HeNe laser (633 nm) and an infrared (IR) laser $(785 \mathrm{~nm})$ and a magnification of $100 x$. The power measured at the sample in both cases was 2-5 $\mu \mathrm{W}$. Raman spectra were recorded on areas of adhesive deposited on glass slides in the cases of Entobdella soleae and E. coli, and directly on amyloid that was deposited onto glass slides from stock solution in the case of the pathogenic amyloid sample. Contamination or artefacts were identified by measuring spectra in different areas and comparing the intensities of the peaks. In this way, the peak at $1580 \mathrm{~cm}^{-1}$ in the spectrum measured with an IR laser and the peak at $1591 \mathrm{~cm}^{-1}$ in the spectrum measured with a HeNe laser could be identified as not belonging to the protein.

\section{Atomic force microscopy (AFM)}

For AFM experiments on $\alpha$-synuclein fibrils, a $20 \mu \mathrm{L}$ drop was deposited onto freshly cleaved mica and allowed to incubate at room temperature for $1 \mathrm{~h}$ to allow sufficient adsorption of the fibrils onto the mica surface. The sample was rinsed gently with milliQ water to remove unbound fibrils and a further $150 \mu \mathrm{L}$ milliQ water added prior to AFM. The fibrils from Entobdella soleae and curli on glass substrates were probed directly in milliQ water. All force curves were obtained with an Asylum MFP3D AFM (Asylum Research, Santa Barbara, CA), using $\mathrm{Si}_{3} \mathrm{~N}_{4}$ cantilevers (CSC38, MikroMasch, Estonia) with calibrated spring constants between 0.5 and $0.8 \mathrm{Nm}^{-1}$. Force measurements were taken with a tip velocity of $1 \mu \mathrm{m} / \mathrm{s}$, and halted at a maximum loading force of 2 $\mathrm{nN}$. Numerous force curves were obtained at different positions on different fibrils. AFM images were taken in intermittent-contact mode before and after force measurements to ensure data was being collected directly on fibrils and that there was no substantial damage or motion of the fibrils caused by the measurements. Image processing and analysis of the force curves, including fitting each force peak with the worm-like chain model in order to calculate parameters such as mean contour length and persistence length, were performed using IGOR PRO (Wavemetrics, OR, USA) data analysis software.

\section{Results and Discussion}

\section{Thioflavin-T binding indicates the presence of amyloid}

In the case of Entobdella soleae, amyloid detection by congo red binding and birefringence is impractical because of the extremely small volume of adhesive produced by the parasite and the problems of harvesting the adhesive. However, direct binding of the adhesive fibres with the fluorochrome dye thioflavin-T was detected with confocal microscopy (Fig. 1). This dye exhibits specific fluorescence when bound to amyloid fibrils (Mostaert et al., 2006), and is commonly used to detect amyloid fibrils, both ex vivo and in vitro. This is supportive, but not conclusive evidence that the adhesive fibrils of Entobdella soleae share structural characteristics with amyloid fibrils.
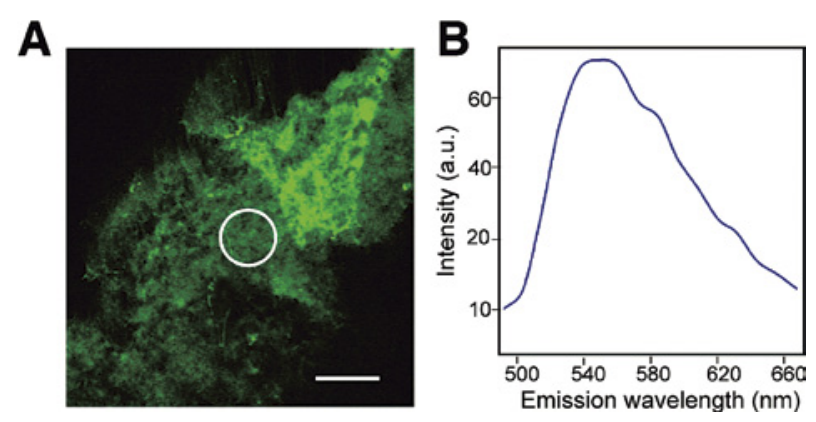

Fig. 1. Direct binding of thioflavin-T to $\beta$-sheet structures within the sectreted adhesive from the marine parasite Entobdella soleae. Thioflavin $\mathrm{T}$ is a benzothiazole dye commonly used to detect amyloid fibrils, both ex vivo and in vitro. A: Confocal microscope image of the secreted adhesive stained with $10 \mu \mathrm{m}$ aqueous thioflavin-T (scale bar, $50 \mu \mathrm{m}$ ). B: Confocal emission spectrum collected for the thioflavin- $\mathrm{T}$ stained adhesive within the region circled in Figure 2A. The spectrum was collected from 505 to $620 \mathrm{~nm}$, after excitation at $488 \mathrm{~nm}$. Emission of thioflavin-T binding to amyloid occurs as a peak around $550 \mathrm{~nm}$ which was determined using stained and unstained control samples of $\mathrm{A} \beta_{25-35}$ amyloid fibrils. 


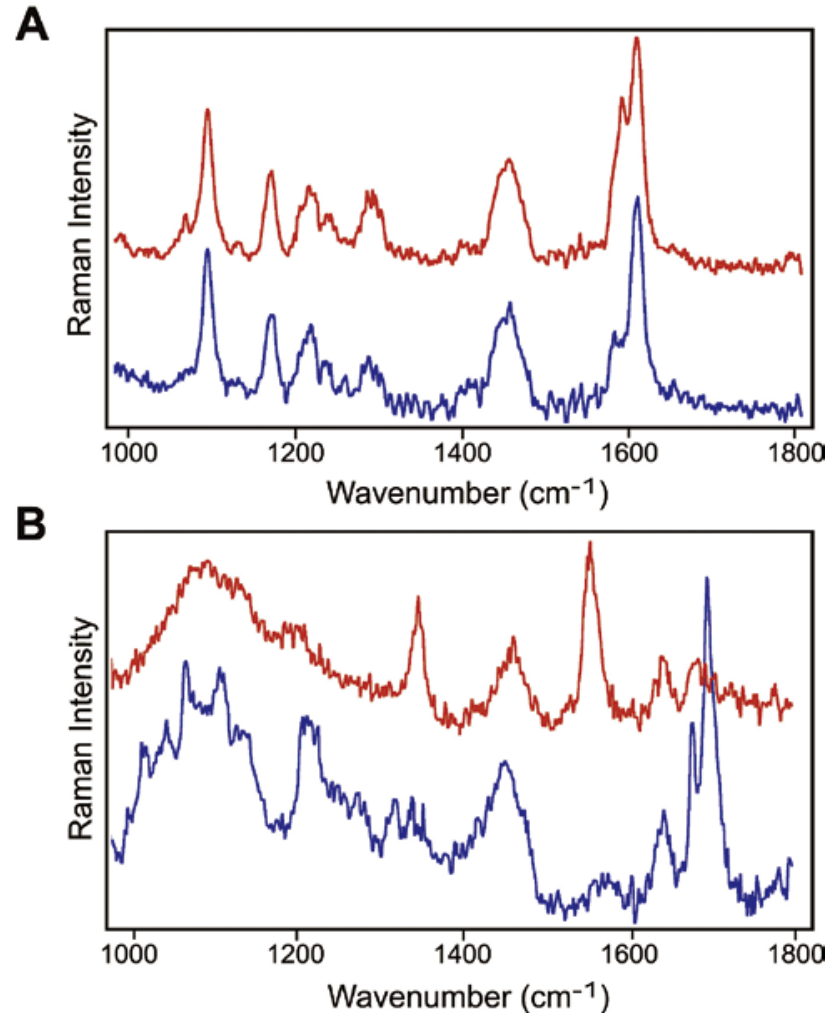

Fig. 2. A: Raman spectra observed for amyloid fibrils in the adhesive extruded from the marine parasite Entobdella soleae. The amide III band was typical for a $\beta$-sheet structure with maxima at 1228 and $1251 \mathrm{~cm}^{-1}$. Attempts to unambiguously assign the amide III bands by exchanging the hydrogen in the peptide backbone with deuterium, were made by incubating for 7 days in $\mathrm{D}_{2} \mathrm{O}$ at $37^{\circ} \mathrm{C}$ (red spectrum), but yielded no significant change in the Raman spectrum (compared to fibrils in $\mathrm{H}_{2} \mathrm{O}$, blue spectrum). B: Raman spectra of the amyloid fibrils produced from $A \beta_{25-35}$ in $\mathrm{H}_{2} \mathrm{O}$ and $\mathrm{D}_{2} \mathrm{O}$. The amide III peaks at 1232 and $1263 \mathrm{~cm}^{-1}$ are not present in the spectrum of the amyloid fibrils grown in $\mathrm{D}_{2} \mathrm{O}$ (red spectrum). As with the protein from Entobdella soleae (Fig. 3A), no significant exchange could be observed in the spectrum of the fibrils grown in $\mathrm{H}_{2} \mathrm{O}$ after incubating in $\mathrm{D}_{2} \mathrm{O}$ (blue spectrum). The Raman spectrum of the amyloid fibrils produced from $\mathrm{A} \beta_{25-35}$ in $\mathrm{H}_{2} \mathrm{O}$ (blue spectrum) contains a very sharp amide I peak at $1667 \mathrm{~cm}^{-1}$ that is only evident as a shoulder of the peak centred at $1650 \mathrm{~cm}^{-1}$ in the spectrum of the fibrils produced in $\mathrm{D}_{2} \mathrm{O}$ (red spectrum).

\section{Raman spectroscopy identifies intermolecular $\beta$-sheets}

To evaluate if the adhesive fibres of Entobdella soleae possess the generic amyloid structure we used Raman spectroscopy to compare the structure of the proteins in the adhesive with amyloid fibrils formed from peptides of A $\beta_{25-35}$ (Fig. 2). This widely studied peptide was chosen due to its ability to form fibrils rapidly in $\mathrm{H}_{2} \mathrm{O}$ (Yankner et al., 1990), providing an avenue for deuterium exchange during the formation process.

In the Raman spectrum $(\mathrm{HeNe}, 633 \mathrm{~nm})$ of $\mathrm{A} \beta_{25-35}$ fibrils, a very sharp amide I peak was prominent at 1667 $\mathrm{cm}^{-1}$ (Fig. 2B, blue). For the adhesive fibrils of Entobdella soleae, a similar peak was observed at $1666 \mathrm{~cm}^{-1}$ (only measurable with an IR laser (785 nm), data not shown). In both cases, the peak was assigned to the $v(0,0)$ mode of the amide I band for an infinite antiparallel $\beta$-sheet. Peaks for the associated $v(\pi, 0)$ mode were lower than expected in both spectra, lying in the region normally associated with phenylalanine, tryptophan or tyrosine. However, the A $\beta_{25-35}$ peptide does not contain any of these amino acids, and thus the peaks centred at 1619 and $1618 \mathrm{~cm}^{-1}$ (Fig. 2B, blue) were unambiguously attributed to the $v(\pi, 0)$ mode. For Entobdella soleae fibrils, the peak at $1612 \mathrm{~cm}^{-1}$ (Fig. 2A, blue) was much too strong to be assigned to these amino acids, that are only present in low concentrations in the secreted adhesive (Hamwood et al., 2002). Thus the peak at $1612 \mathrm{~cm}^{-1}$ was attributed to the $v(\pi, 0)$ mode. The unusually low values for an amide I band are thought to be due to exceptionally strong hydrogen bonding (Barth and Zscherp, 2002). In amyloid fibrils there are extensive intermolecular networks of hydrogen bonding stabilizing the stacks of $\beta$-strands (Sunde and Blake, 1997). For both types of fibril the amide III band was typical for a $\beta$-sheet structure, with maxima at 1228 and $1251 \mathrm{~cm}^{-1}$ (Entobdella soleae, Fig. $2 \mathrm{~A}$, blue), and 1232 and $1263 \mathrm{~cm}^{-1}$ (A $\beta_{25-35}$, Fig. 2B, blue). Attempts were made to unambiguously assign the amide III bands by exchanging the hydrogen with deuterium. However, after 7 days incubation in $\mathrm{D}_{2} \mathrm{O}$ at $37^{\circ} \mathrm{C}$, no significant change was observed in the Raman spectrum of either type of fibril (Fig. 2). We postulate that in both cases the tight, insoluble amyloid fibrils did not allow the $\mathrm{D}_{2} \mathrm{O}$ to penetrate into the structure. For comparison, new fibrils of $\mathrm{A} \beta_{25-35}$ were grown in $\mathrm{D}_{2} \mathrm{O}$ (Fig. 2B, red), and in this case the amide III peaks were not present, confirming the previous attribution of the amide III peaks.

The Raman spectroscopy thus enabled us to establish that the structure of infinite $\beta$-sheets, and exceptionally strong intermolecular hydrogen bonding is common to 


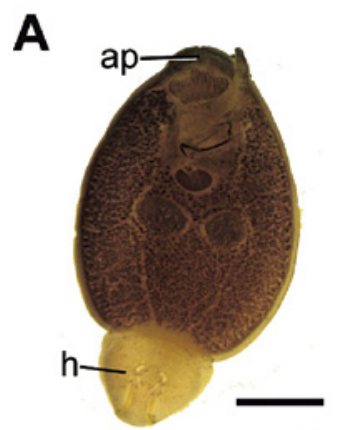

B

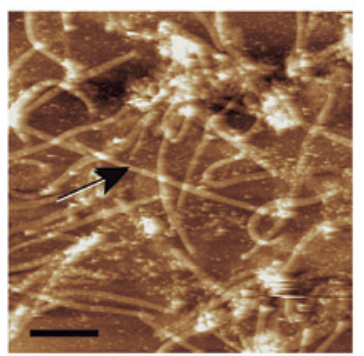

D

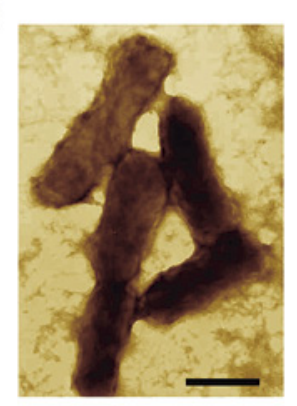

$\mathbf{E}$

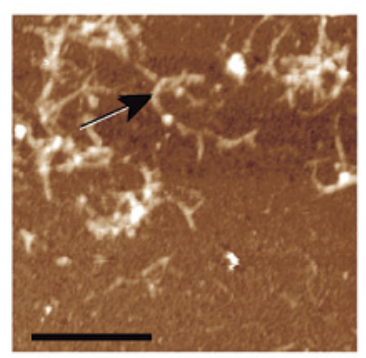

C
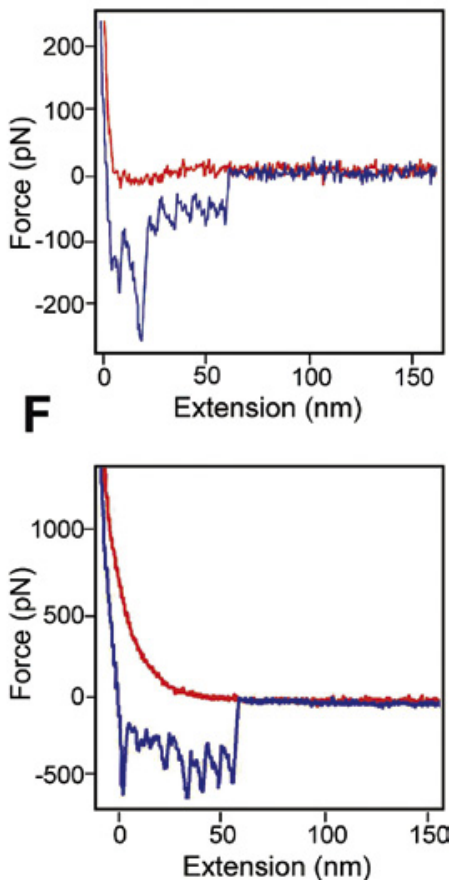

Fig. 3. A: Light micrograph of the marine parasite Entobdella soleae (ventral view). Anterior pads (ap) bind to fish skin using a temporary adhesive secretion, and the haptor (h) attaches to host skin by suction (scale bar, $1 \mathrm{~mm}$ ). B: Atomic Force Microscopy (AFM) image of fibres obtained directly from an adhesive pad print of Entobdella soleae without purification or additional isolation (scale bar, $3 \mu \mathrm{m}$ ). The dark to light height range $=0-15 \mathrm{~nm}$. C: A typical force-extension curve taken from an adhesive fibre of Entobdella soleae (arrow indicates region of fibre where this particular curve was taken). Both the approach (red) and retraction (blue) force traces are shown. D: Transmission electron micrograph of negatively stained ( $0.2 \%$ uranyl acetate) bacteria (E. coli) surrounded by curli fibrils (scale bar, $0.5 \mu \mathrm{m}$ ). E: AFM image of curli fibrils (scale bar, $1 \mu \mathrm{m}$ ). The dark to light height range $=$ $0-10 \mathrm{~nm}$. F: Typical force-extension curve taken for curli fibrils (fibril probed is indicated by the arrow) from the adhesive biofilm of $E$. coli.

both the adhesive fibrils of Entobdella soleae and the amyloid fibrils of $A \beta_{25-35}$.

\section{'Sacrificial bonds' and 'hidden length' provide cohesive strength}

In order to understand how amyloid fibrils function as an adhesive we explored the inelastic response of the fibrils at the molecular level by making nanoscale mechanical measurements using AFM. Measurements were made directly on fibrils extruded from Entobdella soleae (Fig. $3 \mathrm{~A}, \mathrm{~B}$ ) without purification or additional isolation, and curli fibrils (Fig. 3C, D) from E. coli (bacteria surrounded by curli are shown in Fig. 3D). The fibrils were located in AFM imaging mode, and the AFM tip was then brought into contact at a chosen point along the length of a fibril (e.g., as indicated in Fig. 3B, E) to establish non-specific adsorption to the tip (Fukuma et al., 2006). The tip and substrate were then pulled apart under an increasing tensile load with the fibril attached between the two surfaces until the material finally failed and the connection between the tip and substrate, via the fibril, was broken.

Characteristic force versus displacement curves, showing the response of the fibrils on retraction of the AFM tip can be seen in Figure 3C, F. The fibrils display a series of sawtooth mechanical responses reflecting the repetitive breaking of 'sacrificial bonds' 
A

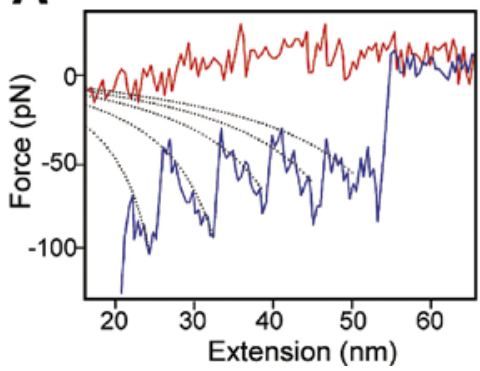

B

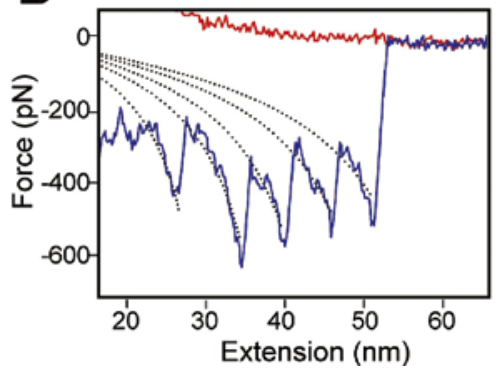

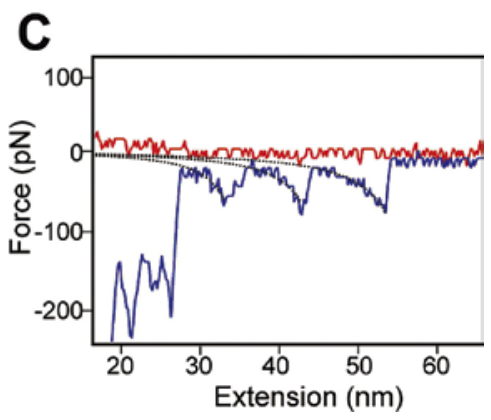

Fig. 4. Comparison of the sawtooth response from amyloid structures using atomic force microscopy. A: Expansion of the sawtooth response in the force-extension curve from the amyloid fibril in the adhesive of Entobdella soleae shown in Figure 1C. B: Sawtooth expansion from the force-extension curve shown in Figure 1F for the curli fibril in the adhesive biofilm of E. coli. C: Expansion of the sawtooth response in a typical force-extension curve from amyloid fibrils grown from $a$-synuclein. Sawtooth responses are shown fit to the WLC model (dotted lines). Both the approach (red) and retraction (blue) force traces are shown.

within an intermolecular $\beta$-sheet as protein is pulled from the surface of the fibril, and the 'hidden length' of the amino-acid chain subsequently extended. This process leads to superior mechanical strength when compared with systems of similar overall length, but without such sacrificial bonds, due to the extra energy required in pulling the material apart (Smith et al., 1999) (corresponding to the area under the curves in the expanded sections shown in Fig. 4A, B). This mechanism of 'hidden length' and 'sacrificial bonds' has been measured previously in modular proteins such as titin (Rief et al., 1997), the natural adhesives of algae (Dugdale et al., 2005; Mostaert et al., 2006), and the 'glue' adding fracture resistance to bone (Fantner et al., 2005). It does not have to be associated with adhesion per se, but instead influences the cohesive strength of the material. When the sawtooth peaks are equally spaced, as in Figure 4 , this indicates an underlying repetitive structural unit within the material.

\section{Worm-like chain fitting reveals differences related to constituent proteins}

By fitting a number of curves using the worm-like chain (WLC) fit for polymers (Bustamante et al., 1994; Rief et al., 1997) we found quantitative variations between the fibrils. In the case of the Entobdella soleae fibrils, a consistent change in contour length of $15.5 \mathrm{~nm}$ (SD $4.2 \mathrm{~nm}, \mathrm{n}=55$ ) was measured. The contour length corresponds to the hidden length which is exposed when sacrificial bonds are broken, and in the case of material composed of protein, the approximate number of amino acids within this hidden length can be found by diving the length by the approximate size of a single stretched amino-acid $(0.36 \mathrm{~nm})$, which in this case gives $\sim 43$ amino acids. The total length of the stretched polypeptide chain is given by the number of amino acids between the two attachment points (in this case the anchor within the intermolecular $\beta$-sheet and the anchor either directly to the tip or to an adjacent fully stretch polypeptide) multiplied by the length of a single stretch amino acid. The hidden length exposed when the protein is pulled from the fibril will always be less than the total length as there is no measure of the initial folded length of the protein within the $\beta$-sheet with this measurement technique (Rief et al. 1997). The number and type of sacrificial bonds cannot be deconvoluted, but the force involved in simultaneously breaking sacrificial bonds corresponds to the peak to trough force, which was found to be $63 \mathrm{pN}$ ( $\mathrm{SD} 40 \mathrm{pN})$.

As can be seen in Figure 4A and B, there is good qualitative agreement between the mechanical response of the adhesive fibrils of Entobdella soleae and curli. In the case of curli, WLC fitting $(n=46)$ revealed a consistent change in contour length of $27.3 \mathrm{~nm}$ (SD 15.2) corresponding to $\sim 76$ amino acids, and peak to trough force of $123 \mathrm{pN}$ (SD $65 \mathrm{pN}$ ). It is interesting to note that approximately twice the number of amino acids are involved in the hidden length of curli, and that this corresponds to twice as much force associated with the breaking of sacrificial bonds. This could be an indication that the magnitude of the individual sacrificial bonds is the same for both systems and relates directly to the number of constituent amino acids involved in 
the intermolecular $\beta$-sheet rather than the nature of the constituent amino acids. Non-specific bonding of this type would be wholly consistent with the generic nature of the amyloid structure and may correspond to the intermolecular hydrogen bonds indicated by Raman spectroscopy. For curli, the primary structural protein is CsgA, consisting of 151 amino acids, 21 of which make up an N-terminal Sec signal sequence and are cleaved off after translocation into the periplasmic space (Wang and Chapman., 2008). Of the remaining 130 amino acids, 109 are proposed to form the amyloid core region containing five imperfect repeating units forming a strand-loopstrand motif (Collinson et al., 1999). If this model is correct then the folded width of the monomer within the amyloid core structure would be approximately $0.5 \mathrm{~nm}$ (width of a $\beta$-strand) $\times 5$ (number of $\beta$-strands) giving a folded width of approximately $2.5 \mathrm{~nm}$. The hidden length exposed as each monomer is removed from the amyloid core structure should then be the total length of the amino acid chain within the core structure $(109 \times 0.36=39.2$ $\mathrm{nm}$ ), minus the folded length of approximately $2.5 \mathrm{~nm}$, which would gives a hidden length of approximately $36.7 \mathrm{~nm}$. Although somewhat higher than our measured value of $27.3 \mathrm{~nm}$ (SD 15.2), this calculated value still lies within the standard deviation of our measurement. In the case of the Entobdella soleae adhesive, the constituent structural protein, or proteins, have yet to be identified, but the above results would indicate that from each constituent protein there are in the region of 60 amino acids associated with the amyloid core structure.

We have shown previously that fibrils formed from short peptide segments are susceptible to damage by the AFM tip during indentation due to the near-crystalline properties of the fibrils (Fukuma et al., 2006). For this reason, the mechanical response of fibrils produced in vitro from wild type $a$-synuclein whole protein was explored for comparison (Fig. 4C). For this 140 aminoacid protein, force curves revealed a change in contour length of $17.0 \mathrm{~nm}(\mathrm{SD} 6.5, \mathrm{n}=20)$, and the peak to trough force was $108.5 \mathrm{pN}$ (SD 35), both values being intermediate between those for curli and the Entobdella soleae fibrils, and much larger than force peaks seen previously for amyloid formed from short peptides (Fukuma et al., 2006). It should be noted that with these fibrils sawtooth responses were rare and, when observed the curves had very few peaks, hence the correspondingly low value of $n$ for these measurements. This may indicate an underlying structural difference between fibrils formed in vitro and those measured ex vivo.

\section{The adhesive component resides at the fibril surface}

In contrast to the adhesive fibrils from the marine parasite and bacterial biofilm, the $\alpha$-synuclein fibrils with no adhesive function show zero separation between the sawtooth response and the base line of zero force in their AFM force curves (Fig. 4C). For the adhesive amyloid fibrils, an initial tensile loading force is required before the structure of the fibrils themselves can be unravelled. This peak to baseline force is $76 \mathrm{pN}$ (SD $46 \mathrm{pN}$ ) for curli, $46 \mathrm{pN}$ (SD $23 \mathrm{pN}$ ) for fibrils of Entobdella soleae, and is likely to be associated with the specific adhesive function of these fibrils (Fig. 4A, B). The force probably serves to unravel an adhesive residue, which does not lie within the intermolecular $\beta$-sheet core structure of the fibril itself but, due to its function, will preferentially bind to the AFM tip. It is only after this residue has been extended that sacrificial bonds within the fibrillar core structure start to be broken and proteins removed. Thus the adhesive component of the material resides at the surface of the fibrils while the cohesive strength of the material exists within the intermolecular $\beta$-sheets making up the amyloid core structure. In the case of curli the glycine rich 21 amino acid residue thought to lie outside the core structure could be responsible for the observed adhesion. The calculated relative toughness for the three types of fibrils (as calculated from the area under the curves without normalizing for the cross-sectional area of the polypeptide chain) was $2.3 \mathrm{aJ}$ (SD $1.8 \mathrm{aJ}$ ) for the adhesive fibrils of Entobdella soleae, 7.2 (SD 4.8) aJ for curli, and $1.2 \mathrm{aJ}$ (SD 0.3) for $\alpha$-synuclein.

\section{Conclusions}

Previously, no mechanistic link between different types of natural adhesive had been identified, and indeed strategies for adhesion were found to differ widely for organisms seeking adhesion in different environments with different mechanical demands placed upon them (Yule and Walker, 1987; Waite, 2002). Consequently, past biomimetic strategies have relied on mimicking the best match for a particular application or combining specific features of different adhesive strategies (Lee et al., 2007). The beauty of the generic amyloid mechanism is that it does not appear to be specific to a particular environment, nor to a specific amino-acid sequence, thus giving it the potential to meet a broad variety of possible adhesive requirements.

While AFM is primarily used for imaging surface structures at the nanoscale, here we have shown that by using the AFM tip to carefully pull apart individual amyloid fibrils we can reveal the underlying core 
structure and relate this to the biological utility of the fibrils in the case of functional amyloid. The molecular level insight provided here should add an additional controllable design parameter at the molecular level, as well as helping to establish any differences between the molecular level structure of functional and pathogenic amyloid fibrils.

\section{Acknowledgements}

We thank Prof. John Pethica, Trinity College Dublin, Ireland and Dr Hugh Byrne, Dublin Institute of Technology, Ireland for fruitful discussions. We also thank Dr Shahin Zibaee, MRC, LMB, Cambridge, UK for providing pre-formed fibrils of recombinant human $\alpha$-synuclein and Simon O’Brien, Trinity College Dublin Ireland for help with data analysis software. This work was funded by Science Foundation Ireland (Grant No. 07/ IN1/B931).

\section{References}

Autumn K, Liang YA, Hsieh ST, Zesch W, Chan WP, Kenny T, Fearing R, Full RJ: Adhesive force of a single gecko foot-hair. Nature 405: 681-685 (2000).

Autumn K, Sitti M, Liang YA, Peattie AM, Hansen WR, Sponberg S, Kenny TW, Fearing R, Israelachvili JN, Fuji RJ: Evidence for van der Waals adhesion in gecko setae. Proc Natl Acad Sci USA 99: 12252-12256 (2002).

Barth A, Zscherp C: What vibrations tell us about proteins. Q Rev Biophys 35: 369-430 (2002).

Bustamante C, Marko JF, Siggia ED, Smith S: Entropic elasticity of lambda-phage DNA. Science 265: 1599600 (1994).

Chapman MR, Robinson LS, Pinkner JS, Roth R, Heuser J, Hammar M, Normark S, Hultgren SJ: Role of Escherichia coli curli operons in directing amyloid fiber formation. Science 295: 851-855 (2002).

Cherny I, Gazit E: Amyloids: Not only pathological agents but also ordered nanomaterials. Angew Chem Int Ed 47: 2-10 (2008).

Chiti F, Dobson CM: Protein misfolding, functional amyloid, and human disease. Annu Rev Biochem 75: 333-366 (2006).

Collinson SK, Parker JMR, Hodges RS, Kay WW: Structural predictions of AgfA, the insoluble fimbrial subunit of salmonella thin aggregative fimbriae. J Mol Biol 290: 741-756 (1999).
Dugdale TM, Dagastine R, Chiovitti A, Mulvaney P, Wetherbee R: Single adhesive nanofibers from a live diatom have the signature fingerprint of modular proteins. Biophys J 89: 4252-4260 (2005).

Fantner GE, Hassekan T, Kindt JH, Weaver JC, Birkedal H, Pechenik L, Cutroni JA, Cidade GA, Stucki GD, Morse DE, Hansma PK:Sacrificial bonds and hidden Sacrificial bonds and hidden length dissipate energy as mineralized fibrils separate during bone fracture. Nature Mater 4: 612-616 (2005).

Fowler DM, Koulov AV, Balch WE, Kelly JW: Functional amyloid - from bacteria to humans. Trends Biochem Sci 32: 217-224 (2007).

Fukuma T, Mostaert AS, Jarvis SP: Explanation for the mechanical strength of amyloid fibrils. Tribol Lett 22: 233-237 (2006).

Hamwood TE, Cribb BW, Halliday JA, Kearn GC, Whittington ID: Preliminary characterisation and extraction of anterior adhesive secretion in monogenean (platyhelminth) parasites. Folia Parasitol 49: 39-49 (2002).

Kamino K: Underwater adhesive of marine organisms as the vital link between biological science and materials science. Mar Biotechnol 10: 111-121 (2008).

Kearn GC, Evans-Gowing R: Attachment and detachment of the anterior adhesive pads of the monogenean (platyhelminth) parasite Entobdella soleae from the skin of the common sole (Solea solea). Int J Parasitol 28: 1583-1593 (1998).

Lansbury PT: Evolution of amyloid: what normal protein folding may tell us about fibrillogenesis and disease. Proc Natl Acad Sci USA 96: 3342-3344 (1999).

Larsen P, Nielsen JL, Dueholm MS, Wetzel R, Otzen D, Nielsen PH: Amyloid adhesions are abundant in natural biofilms. Environ Microbiol 9: 3077-3090 (2007).

Lee H, Lee BP, Messersmith PB: A reversible wet/dry adhesive inspired by mussels and geckos. Nature 448: 338-341 (2007).

Mostaert AS, Higgins MJ, Fukuma T, Rindi F, Jarvis SP: Nanoscale mechanical characterisation of amyloid fibrils discovered in a natural adhesive. J Biol Phys 32: 393-401 (2006).

Olsen A, Jonsson A, Normark S: Fibronectin binding mediated by a novel class of surface organelles on Escherichia coli. Nature 338: 652-655 (1989).

Otzen D, Nielsen PH: We find the here, we find them there: Functional bacterial amyloid. Cell Mol Life Sci 65: 910-927 (2007).

Pawar DM, Rossman ML, Chen J: Role of curli fimbriae in mediating the cells of enterohaemorrhagic Escherichia coli to attach to abiotic surfaces. J Appl Microbiol 99: 418-425 (2005). 
Rief M, Gautel M, Oesterhelt F, Fernandez JM, Gaub HE: Reversible unfolding of individual titin immunoglobulin domains by AFM. Science 76: 11091112 (1997).

Robinson LS, Ashman EM, Hultgren SJ, Chapman MR: Secretion of curli fibre subunits is mediated by the outer membrane-localized CsgG protein. Mol Microbiol 59: 870-881 (2006).

Smith BL, Schäffer TE, Viani M, Thompson JB, Frederick NA, Kindt J, Belcher A, Stucky GD, Morse DE, Hansma PK: Molecular mechanistic origin of the toughness of natural adhesives, fibres and composites. Nature 399: 761-763 (1999).

Sunde M, Blake C: The structure of amyloid fibrils by electron microscopy and X-ray diffraction. Adv Protein Chem 50: 123-159 (1997).

Waite JH: Adhesion in byssally attached bivalves. Biol Rev 58: 209-231 (1983).

Waite JH: Adhesion à la moule. Integr Comp Biol 42, 1172-1180 (2002).
Waite JH, Tanzer ML: Phenolic substance of Mytilus edulis: Novel adhesive containing L-dopa and hydroxyproline. Science 212: 1038-1040 (1981).

Walker G: Marine organisms and their adhesion. In: Synthetic Adhesives and Sealants, Critical Reports on Applied Chemistry (Wake WC, ed), John Wiley and Sons, Chichester, 1987 (p. 112-135).

Wang X, Chapman MR: Sequence determinants of bacterial amyloid formation. J Mol Biol 380: 570-580 (2008).

Yankner BA, Duffy LK, Kirschner DA: Neurotrophic and neurotoxic effects of amyloid beta protein: reversal by tachykinin neuropeptides. Science 250: 279-282 (1990).

Yule AB, Walker G: Adhesion in barnacles. In: Barnacle Biology Crustacean Issues 5 (Southward A, ed), A. A. Balkema, Rotterdam, 1987 (p. 389-402).

Zibaee S, Jakes R, Fraser G, Serpell LC, Crowther RA, Goedert M: Sequence determinants for amyloid fibrillogenesis of human $\alpha$-synuclein. J Mol Biol 374: 454-464 (2007). 